PROCEEDINGS OF THE

AMERICAN MATHEMATICAL SOCIETY

Volume 133, Number 6, Pages 1587-1591

S 0002-9939(04)07673-7

Article electronically published on December 6, 2004

\title{
SOLUTIONS TO TWO QUESTIONS ABOUT THE WEYL ALGEBRAS
}

\author{
V. BAVULA
}

(Communicated by Martin Lorenz)

\begin{abstract}
Affirmative answers are given to the following two questions about the Weyl algebras: a question of J. Alev: Does the first Weyl algebra contain a non-noetherian subalgebra?, and a question of T. Lenagan: Is there a uniserial module $M$ of length 2 over the Weyl algebra $A_{n}$ with a holonomic submodule $U$ such that $V=M / U$ is non-holonomic?
\end{abstract}

\section{The first Weyl algebra contains a Left And Right NON-NOETHERIAN SUBALGEBRA}

Let $K$ be a field of characteristic zero. The (first) Weyl algebra $A_{1}=\langle x, \partial| \partial x-$ $x \partial=1\rangle$ is a simple noetherian domain. Any commutative subalgebra of the Weyl algebra $A_{1}$ is a finitely generated algebra (hence noetherian) 1, 7]. An old problem of Dixmier [7] which is still open (problem 4, p. 242) asks whether for each $K$-derivation $\delta$ of the Weyl algebra $A_{1}$ the commutative graded algebra $\operatorname{gr}(N(\delta)):=$ $\bigoplus_{i \geq 0} \operatorname{ker}\left(\delta^{i}\right) / \operatorname{ker}\left(\delta^{i-1}\right)$ is finitely generated (an affirmative answer to this problem would imply that the filtered subalgebra $N(\delta)=\bigcup_{i \geq 0} \operatorname{ker}\left(\delta^{i}\right)$ of the Weyl algebra $A_{1}$ is a finitely generated noetherian algebra). Many ring theorists (including the author) believe that the answer is affirmative. Numerous 'experimental facts' support this belief.

For quite some time the following question of J. Alev was open: Does the first Weyl algebra contain a non-noetherian subalgebra? Lemma 1.1 gives an example of such a subalgebra.

Let $B:=K[h][x ; \sigma]$ be a skew polynomial algebra with coefficients from a polynomial algebra $K[h]$ in one variable $h$ where the $K$-algebra automorphism $\sigma$ of $K[h]$ is given by the rule $\sigma(h)=h-1$. So, $B=\bigoplus_{i \geq 0} K[h] x^{i}$ and

$$
a(h) x^{i} b(h) x^{j}=a(h) \sigma^{i}(b(h)) x^{i+j}=a(h) b(h-i) x^{i+j}
$$

for all $i, j \geq 0$, where $a(h), b(h) \in K[h]$.

The algebra $B$ is a subalgebra of the Weyl algebra $A_{1}$ via the algebra monomorphism

$$
B \rightarrow A_{1}, x \mapsto x, h \mapsto \partial x
$$

Received by the editors December 20, 2003 and, in revised form, February 13, 2004.

2000 Mathematics Subject Classification. Primary 16S32, 16P40, 16W50, 16W70.

(C)2004 American Mathematical Society 
For each $i \geq 1$, let $K[h]_{<i}:=\{a \in K[h] \mid \operatorname{deg}(a)<i\}$ and $K[h]_{<0}:=K$. Obviously, $\sigma\left(K[h]_{<i}\right)=K[h]_{<i}$ for all $i \geq 0$. It follows that $C:=\bigoplus_{i \geq 0} K[h]_{<i} x^{i}$ is a subalgebra of $B$.

Lemma 1.1. The subalgebra $C$ of the Weyl algebra $A_{1}$ is not a finitely generated algebra and is not a left and right noetherian algebra.

Proof. The algebra $C=\bigoplus_{i \geq 0} C_{i}$ is a positively graded algebra where $C_{i}:=$ $K[h]_{<i} x^{i}$ with

$$
\begin{aligned}
C_{i} C_{j}=K[h]_{<i} \sigma^{i}\left(K[h]_{<j}\right) x^{i+j} & \subseteq K[h]_{<i} K[h]_{<j} x^{i+j} \\
& \subseteq K[h]_{<i+j-1} x^{i+j} \subset K[h]_{<i+j} x^{i+j}=C_{i+j},
\end{aligned}
$$

a strict inclusion for all $i, j \geq 1$. It follows that the algebra $C$ is not finitely generated and that the ideal $I:=\bigoplus_{i>1} C_{i}$ of the algebra $C$ is not a finitely generated left (or right) $C$-module. This means that $C$ is not a left (or right) noetherian algebra.

\section{Non-holonomic modules of Length two over the Weyl algebra $A_{n}$}

Let $K$ be a field of characteristic zero. The Weyl algebra $A_{n}=A_{n}(K)$ is a $K$ algebra generated over the field $K$ by $2 n$ generators $x_{1}, \ldots, x_{n}, \partial_{1}, \ldots, \partial_{n}$ subject to the defining relations:

$$
\partial_{i} x_{j}-x_{j} \partial_{i}=\delta_{i j} \text { (the Kronecker delta), } x_{i} x_{j}=x_{j} x_{i}, \partial_{i} \partial_{j}=\partial_{j} \partial_{i} .
$$

The Weyl algebra $A_{n}$ is canonically isomorphic to the ring of differential operators with polynomial coefficients $K\left[x_{1}, \ldots, x_{n}, \frac{\partial}{\partial x_{1}}, \ldots, \frac{\partial}{\partial x_{n}}\right]\left(x_{i} \leftrightarrow x_{i}, \partial_{i} \leftrightarrow \frac{\partial}{\partial x_{i}}\right)$.

In this section, $K=\mathbb{C}$ is the field of complex numbers. The Weyl algebra $A_{n}=$ $A_{n}(\mathbb{C})$ is a simple Noetherian domain of Gelfand-Kirillov dimension GK $\left(A_{n}\right)=2 n$, $A_{n}=A_{1} \otimes \cdots \otimes A_{1}, n$ times. J. Bernstein proved that GK $(M) \geq n$ for any nonzero finitely generated $A_{n}$-module (44 or [5], Chap. 1).

A finitely generated $A_{n}$-module $M$ is called holonomic if $\operatorname{GK}(M)=n$. In the case of the first Weyl algebra $A_{1}$ all simple modules were classified by R. Block, [] (a different approach is given in [3]) and all of them are holonomic $A_{1}$-modules. Let $M_{1}, \ldots, M_{n}$ be nonzero simple $A_{1}$-modules. Then their tensor product $\bigotimes_{i=1}^{n} M_{i}$ is a simple holonomic $A_{n}$-module. It was believed that all simple $A_{n}$-modules $(n \geq 2)$ are holonomic, but T. Stafford gave an example of a simple non-holonomic $A_{n}$-module, [10].

Theorem 2.1 (Theorem 1.1, [10]). For $2 \leq i \leq n$, pick $\lambda_{i} \in \mathbb{C}$ that are linearly independent over the field of rational numbers $\mathbb{Q}$. Then the element

$$
\alpha=x_{1}+\left(\sum_{2}^{n} \lambda_{i} \partial_{i} x_{i}\right) \partial_{1}+\sum_{2}^{n}\left(x_{i}+\partial_{i}\right)
$$

generates a maximal left ideal of $A_{n}$. In particular, the simple $A_{n}$-module $A_{n} / A_{n} \alpha$ has Gelfand-Kirillov dimension $2 n-1$.

Denote by $S t_{n}(\alpha)$ the Stafford's module $A_{n} / A_{n} \alpha$ from Theorem 2.1

Question of Lenagan ([8]). Is there a uniserial module $M$ of length 2 over the Weyl algebra $A_{n}$ with a holonomic submodule $U$ such that $V=M / U$ is nonholonomic. 
Theorem 2.2 gives a positive answer to this question.

Given $\mu \in \mathbb{C} \backslash \mathbb{Z}$, then the $A_{1}$-module

$$
S(\mu)=A_{1} / A_{1}\left(\partial_{1} x_{1}-\mu\right)
$$

is simple, and two such modules are isomorphic, i.e., $S(\mu) \simeq S\left(\mu^{\prime}\right)$, iff $\mu-\mu^{\prime} \in \mathbb{Z}$ (3], Theorem 3.2).

Then the tensor product of the modules above

$$
S\left(\mu_{1}, \ldots, \mu_{n}\right)=\bigotimes_{1}^{n} S\left(\mu_{i}\right) \simeq A_{n} / A_{n}\left(\partial_{1} x_{1}-\mu_{1}, \ldots, \partial_{n} x_{n}-\mu_{n}\right)
$$

is a simple holonomic $A_{n}$-module and two such modules are isomorphic if and only if the corresponding tensor multiples are isomorphic as $A_{1}$-modules (that is, $\mu_{i}-\mu_{j} \in \mathbb{Z}$ for all $\left.i, j\right)$.

Theorem 2.2. Let the $A_{n}$-modules $S t_{n}(\alpha)$ and $S\left(\mu_{1}, \ldots, \mu_{n}\right)$ be as above $(n \geq 2)$. Then

$$
\operatorname{dim} \operatorname{Ext}_{A_{n}}^{1}\left(S t_{n}(\alpha), S\left(\mu_{1}, \ldots, \mu_{n}\right)\right)=\infty .
$$

Remark. G. Perets 9] gave an example of a uniserial module $M$ of length 2 over the Weyl algebra $A_{n}$ with a non-holonomic submodule $U$ such that $V=M / U$ is holonomic.

2.1. Proof of Theorem 2.2 Let $D$ be a ring with an automorphism $\sigma$ and a central element $a$. The generalized Weyl algebra $A=D(\sigma, a)$ of degree 1 is the ring generated by $D$ and two indeterminates $X$ and $Y$ subject to the defining relations [2]:

$$
X \alpha=\sigma(\alpha) X \text { and } Y \alpha=\sigma^{-1}(\alpha) Y, \forall \alpha \in D, Y X=a \text { and } X Y=\sigma(a) .
$$

The algebra $A=\bigoplus_{n \in \mathbb{Z}} A_{n}$ is a $\mathbb{Z}$-graded algebra where $A_{n}=D v_{n}, v_{n}=X^{n}$ $(n>0), v_{n}=Y^{-n}(n<0)$, and $v_{0}=1$. It follows from the defining relations that

$$
v_{n} v_{m}=(n, m) v_{n+m}
$$

for some $(n, m) \in D$.

The first Weyl algebra is a generalized Weyl algebra

$$
A_{1} \simeq K[H](\sigma, a=H), \quad x \leftrightarrow X, \partial \leftrightarrow Y, \quad \partial x \leftrightarrow H,
$$

with $D=K[H]$, the polynomial algebra in one variable, and $\sigma: H \rightarrow H-1$. We say that an element

$$
u=d_{i} v_{i}+d_{i+1} v_{i+1}+\cdots+d_{j} v_{j} \in A_{1}, \text { all } d_{k} \in D, \quad d_{i} \neq 0, d_{j} \neq 0,
$$

has length $l(u)=j-i$. Clearly, $l(u v)=l(u)+l(v)$ for $u, v \in A_{1}$.

For $\mathbf{k}:=\left(k_{1}, \ldots, k_{n}\right) \in \mathbb{Z}^{n}$, let $v_{\mathbf{k}}=v_{k_{1}}(1) \cdots v_{k_{n}}(n)$ where for $1 \leq i \leq n$ and $m \geq 0: v_{m}(i)=\left(x_{i}\right)^{m}, v_{-m}(i)=\left(\partial_{i}\right)^{m}, v_{0}(i)=1$. The Weyl algebra $\overline{A_{n}}$ is the tensor product of the first Weyl algebras $A_{1}^{(i)}=\mathbb{C}\left\langle x_{i}, \partial_{i}\right\rangle=\mathbb{C}\left[H_{i}\right]\left(\sigma_{i}, H_{i}\right)$. It follows from the definition of generalized Weyl algebras that

$$
A_{n}=\bigoplus_{\mathbf{k} \in \mathbb{Z}^{n}} A_{\mathbf{k}}
$$

is a $\mathbb{Z}^{n}$-graded algebra $\left(A_{\mathbf{k}} A_{\mathbf{e}} \subset A_{\mathbf{k}+\mathbf{e}}, \forall \mathbf{k}, \mathbf{e} \in \mathbb{Z}^{n}\right)$ where $A_{\mathbf{k}}=K\left[H_{1}, \ldots, H_{n}\right] v_{\mathbf{k}}$ and $K\left[H_{1}, \ldots, H_{n}\right]$ is the polynomial algebra in $n$ variables. The $\mathbb{Z}^{n}$-grading above of the Weyl algebra $A_{n}$ is the tensor product of the $\mathbb{Z}$-gradings of the tensor multiples $A_{1}^{(i)}$. 
The $A_{1}$-module $S(\mu)(\mu \notin \mathbb{Z})$ is a $\mathbb{Z}$-graded module:

$$
\begin{aligned}
S(\mu) & =\bigoplus_{i \in \mathbb{Z}} S(\mu)_{i}, \quad S(\mu)_{i}=\mathbb{C} e_{i}, \quad e_{i}=v_{i}+A_{1}(H-\mu), \\
H e_{i} & =(\mu+i) e_{i}, \quad X e_{k}=e_{k+1}, \quad Y e_{-k}=e_{-k-1} \quad(k \geq 0), \\
X e_{-m} & =(\mu-m) e_{-m+1}, \quad Y e_{m}=(\mu+m-1) e_{m-1} \quad(m>0) .
\end{aligned}
$$

We say that a nonzero element

$$
s=s_{i} e_{i}+s_{i+1} e_{i+1}+\cdots+s_{j} e_{j} \in S(\mu), \text { all } s_{k} \in \mathbb{C}, \quad s_{i} \neq 0, s_{j} \neq 0,
$$

has length $l(s)=j-i$. Then $l(u s)=l(u)+l(s)$ for $s \in S(\mu)$ and $u=d_{m} v_{m}+$ $\cdots+d_{n} v_{n} \in A_{1}$ such that none of the scalars from the set $\mu+\mathbb{Z}$ is a root of the polynomial $d_{m} d_{n} \neq 0$. $\mathbb{Z})$ :

Consider the tensor product of the $A_{1}$-modules $S\left(\mu_{i}\right), i=1, \ldots, n$, above $\left(\mu_{i} \notin\right.$

$$
S=S\left(\mu_{1}, \ldots, \mu_{n}\right)=\bigotimes_{i=1}^{n} S\left(\mu_{i}\right) .
$$

It is a $\mathbb{Z}^{n}$-graded $A_{n}$-module

$$
S=\bigoplus_{\mathbf{k} \in \mathbb{Z}^{n}} S_{\mathbf{k}}, \quad S_{\mathbf{k}}=S\left(\mu_{1}\right)_{k_{1}} \otimes \cdots \otimes S\left(\mu_{n}\right)_{k_{n}}, \quad \mathbf{k}=\left(k_{1}, \ldots, k_{n}\right),
$$

and a $\mathbb{Z}$-graded $A_{n}$-module with respect to the total $\mathbb{Z}$-grading

$$
S=\bigoplus_{j \in \mathbb{Z}} S_{j}, \quad S_{j}=\bigoplus\left\{\bigotimes_{i=1}^{n} S\left(\mu_{i}\right)_{m_{i}} \mid m_{1}+\cdots+m_{n}=j\right\} .
$$

Fix $k \in\{1, \ldots, n\}$. A nonzero element $s \in S$ can be uniquely written as a sum

$$
s=s_{i} e_{i}(k)+s_{i+1} e_{i+1}(k)+\cdots+s_{j} e_{j}(k), \quad s_{i} \neq 0, \quad s_{j} \neq 0,
$$

where the coefficients $s_{t}$ are from the tensor product $\bigotimes_{m \neq k} S\left(\mu_{m}\right)$ and $\left\{e_{i}(k)\right\}$ is the basis of the $A_{1}$-module $S\left(\mu_{k}\right)$. The $k$-length of the element $s$ is defined as $l_{k}(s)=j-i$.

Let $\alpha$ be from Theorem 2.1 and $0 \neq s \in S$. Now it is easy to see that

$$
l_{k}(\alpha s)=l_{k}(\alpha)+l_{k}(s)=2+l_{k}(s) \geq 2 \text {, for any } k>1 .
$$

So, the linear map

$$
\alpha_{S}: S \rightarrow S, s \rightarrow \alpha s,
$$

is injective and the cokernel $\operatorname{coker}\left(\alpha_{S}\right) \equiv S / \alpha S$ of the map $\alpha_{S}$ has

$$
\operatorname{dim} \operatorname{coker}\left(\alpha_{S}\right)=\infty
$$

since $\operatorname{im}\left(\alpha_{S}\right) \cap W=0$ where $W=S\left(\mu_{1}\right) \otimes \cdots \otimes S\left(\mu_{k-1}\right) \otimes \overline{1} \otimes S\left(\mu_{k+1}\right) \otimes \cdots \otimes S\left(\mu_{n}\right)$, $\overline{1}=1+A_{1}\left(H_{k}-\mu_{k}\right)$, and $\operatorname{dim}(W)=\infty$.

Using the short exact sequence

$$
0 \rightarrow A_{n} \rightarrow^{\cdot \alpha} A_{n} \rightarrow S t(\alpha) \rightarrow 0,
$$

we get $\operatorname{Ext}_{A_{n}}^{1}\left(S t_{n}(\alpha), S\right)=\operatorname{coker}\left(\alpha_{S}\right)$; hence, by (2) $\operatorname{dim}_{\operatorname{Ext}_{A_{n}}^{1}}^{1}\left(S t_{n}(\alpha), S\right)=\infty$. This finishes the proof of Theorem 2.2

For a polynomial $f \in K\left[H_{1}, \ldots, H_{n}\right]$ let $V(f)$ be the set

$$
\left\{\left(\lambda_{1}, \ldots \lambda_{n}\right) \in \mathbb{C}^{n}: f\left(\lambda_{1}, \ldots \lambda_{n}\right)\right\}=0 .
$$


Corollary 2.3. Suppose that $u=\sum_{\mathbf{i} \in \mathbb{Z}^{n}} u_{\mathbf{i}} v_{\mathbf{i}} \in A_{n}$ is not a homogeneous element where all $u_{\mathbf{i}} \in K\left[H_{1}, \ldots, H_{n}\right]$, the $A_{n}$-module $S=\bigotimes_{i=1}^{n} S\left(\mu_{i}\right)$ is as above (i.e. all $\left.\mu_{i} \in \mathbb{C} \backslash \mathbb{Z}\right)$ and

$$
\left\{\mathbb{Z}^{n} \cup\left(\left(\mu_{1}, \ldots, \mu_{n}\right)+\mathbb{Z}^{n}\right)\right\} \cap V\left(u_{\mathbf{i}}\right)=\emptyset, \text { for all } \mathbf{i} .
$$

Then the linear map $u_{S}: S \rightarrow S, s \rightarrow u s$, is injective and $\operatorname{dim} \operatorname{coker}\left(u_{S}\right)=\infty$.

Proof. The element $u$ is not homogeneous, so there exists $k$ such that $l_{k}(u) \geq 1$. Now, by (3),

$$
l_{k}(u s)=l_{k}(u)+l_{k}(s)>0 \text { for any } 0 \neq s \in S .
$$

So, the linear map $u_{S}$ is injective and $\operatorname{im}\left(u_{S}\right) \cap W=0$ where $W$ is as above. Hence $\operatorname{dim} \operatorname{coker}\left(u_{S}\right)=\infty$.

\section{REFERENCES}

[1] S. A. Amitsur, Commutative linear differential operators, Pacific J. Math. 8 (1958), 1-10. MR.0095305 (20:1808)

[2] V. V. Bavula, The finite-dimensionality of $\mathrm{Ext}^{n}{ }^{n}$ 's and $\mathrm{Tor}_{n}$ 's of simple modules over a class of algebras, Funct. Anal. and Appl. 25 (1991), no. 3, 80-82. MF 1139880 (92k:16019)

[3] V. V. Bavula, Generalized Weyl algebras and their representations, Algebra i Analiz, 4 (1992), no. 1, 75-97; English trans. in St.Petersburg Math. J. 4 (1993), no. 1, 71-92. MR1171955 (93h:16043)

[4] I. N. Bernstein, Modules over a ring of differential operators. An investigation of the fundamental solutions of equations with constant coefficients. (Russian) Funkcional. Anal. $i$ Prilozen. 5 (1971) no. 2, 1-16. MR0290097|(44:7282)

[5] J.-E. Björk, Rings of differential operators. North Holland, Amsterdam, 1979. MR0549189 (82g:32013)

[6] R. E. Block, The irreducible representations of the Lie algebra $s l(2)$ and of the Weyl algebra, Adv. Math. 39 (1981), 69-110. MR0605353 (83c:17010)

[7] J. Dixmier, Sur les algèbres de Weyl, Bull. Soc. Math. France 96 (1968), 209-242. MR0242897 (39:4224)

[8] T. H. Lenagan, Stability of Gelfand-Kirillov dimension for rings with the strong second layer condition, Proc. Edinburgh Math. Soc. 37 (1994), 347-354. MR1280687 (95g:16019)

[9] G. S. Perets, $d$-critical modules of length 2 over Weyl algebras, Israel J. of Math. 83 (1993), 361-368. MR 1239069 (94m:17007)

[10] J. T. Stafford, Nonholonomic modules over Weyl algebras and enveloping algebras, Invent. Math. 79 (1985), 619-638. MR0782240 (86h:17009)

Department of Pure Mathematics, University of Sheffield, Hicks Building, Sheffield S3 7RH, ENGLAND

E-mail address: v.bavula@sheffield.ac.uk 